\title{
Adult visual experience promotes recovery of primary visual cortex from long-term monocular deprivation
}

\author{
Quentin S. Fischer, ${ }^{1,4}$ Salman Aleem, ${ }^{2}$ Hongyi Zhou, ${ }^{2}$ and Tony A. Pham ${ }^{1,2,3,4}$ \\ ${ }^{1}$ Department of Psychiatry and Behavioral Sciences, Baylor College of Medicine, Houston Texas 77030, USA; ${ }^{2}$ Department of \\ Molecular and Cellular Biology, Baylor College of Medicine, Houston Texas 77030, USA; ${ }^{3}$ Department of Neuroscience, Baylor \\ College of Medicine, Houston Texas 77030, USA
}

\begin{abstract}
Prolonged visual deprivation from early childhood to maturity is believed to cause permanent visual impairment. However, there have been case reports of substantial improvement of binocular vision in human adults following lifelong visual impairment or deprivation. These observations, together with recent findings of adult ocular dominance plasticity in rodents, led us to re-examine whether adult primary visual cortex (VI) is capable of any recovery following long-term monocular deprivation starting in development. Using mice as a model, we find that monocular deprivation from early development to mature ages (well past the critical period) severely impaired binocular vision by reducing the amplitude of responses elicited by stimulation of the deprived eye. Surprisingly, we find little effect on nondeprived eye responses. Restoration of binocular vision in mature adults yields modest but significant improvement of visual responses in V1. Remarkably, we find that when binocular vision is followed by occlusion of the nondeprived eye, visual responses in V1 recover almost fully, as measured by visual evoked potential amplitude, spatial frequency threshold, and single-unit activity. We conclude that adult V1 can recover from long-term deprivation when provided with an optimal regimen of visual experience.
\end{abstract}

In comparison to the brain of children, the adult brain is thought to have a more limited capacity for regeneration and repair. One example of the decline of plasticity with age is the effect of monocular deprivation (MD). Classical electrophysiological studies found that early in life even a brief period of MD caused the visual cortex to respond very poorly to the deprived eye (in cats: Wiesel and Hubel 1963; Hubel and Wiesel 1970; Olson and Freeman 1975; Freeman and Olson 1982; in monkeys: Blakemore et al. 1978, 1981; in rodents: Fagiolini et al. 1994; Gordon and Stryker 1996). Restoration of vision to the deprived eye can reverse the effect of MD only if the deprivation was brief. No recovery was observed after prolonged deprivation spanning a critical period of development (in cats: Wiesel and Hubel 1965; Hubel and Wiesel 1970; Blakemore and Van Sluyters 1974; in monkeys: Blakemore et al. 1978). These studies gave rise to the concept that disorders of central circuit development must be diagnosed and treated very early in childhood.

Yet the adult brain appears to be more resilient and plastic than previously believed. A growing number of studies have found morphological and functional changes in motor and sensory neocortex as a result of altered experience or injury in adulthood (Merzenich et al. 1984; Jenkins et al. 1990; Allard et al. 1991; Fox 1992, 1994; Skibinska et al. 2000; Qi et al. 2000; Kaas and Qi 2004). Even in V1, our laboratory and others have found that ocular dominance (OD) plasticity exists in adult mice and rats (Guire et al. 1999; Sawtell et al. 2003; Pham et al. 2004; Lickey et al. 2004; Tagawa et al. 2005; Hofer et al. 2006; Fischer et al. 2007). These findings suggest the possibility that the effects of

${ }^{4}$ Corresponding authors.

E-mail qfischer@bcm.edu; fax (713) 798-8005.

E-mail tapham@bcm.edu; fax (713) 798-8005.

Article is online at http://www.learnmem.org/cgi/doi/10.1101//m.676707. prolonged deprivation imposed early in life can be ameliorated by experience in adulthood. Supporting this contention, a recent multicenter clinical trial involving amblyopic adolescents aged 13-17 yr showed that significant visual improvement was possible with visual therapy well beyond the closure of the "critical period" ( $~ 8 \mathrm{yr}$ of age) (Scheiman et al. 2005). Also, adults with amblyopia can improve their visual performance by visual training (Levi and Polat 1996; Polat et al. 2004; Levi 2005). But the neural substrates mediating this adult plasticity are unknown. It is unclear whether changes in adult vision are mediated by changes in V1 or whether they are mediated at cortical levels beyond $\mathrm{V} 1$.

Thus, using electrophysiological methods that can detect adult OD plasticity, we have re-examined the issue of whether adult V1 can recover from prolonged MD. We subjected mice to MD from shortly after eye opening until mature ages well past the critical period. We find that reopening the deprived eye following this prolonged deprivation produced only partial recovery of visual function. However, binocular experience (BE) followed by suture of the originally nondeprived eye (reverse suture, RS) produced nearly complete recovery of both OD and visual acuity even in mature adults. Our findings suggest that adult visual experience can restore visual functions which fail to develop properly as a result of deprivation in early development.

\section{Results}

BE alone produces only partial recovery

$\mathrm{BE}$ and RS are two traditional paradigms for promoting visual recovery from $\mathrm{MD}$. BE involves simply reopening the deprived eye, whereas RS entails opening the deprived eye and simultaneously suturing shut the nondeprived eye. Recent studies of young cats during the critical period have indicated that BE may 
promote faster recovery from short-term MD than does RS (Mitchell et al. 2001; Kind et al. 2002).

We therefore examined the efficacy of BE in facilitating recovery of mouse V1 from long-term MD (LTMD). In all experiments described in this article, mice were housed in standard transparent plastic cages (see Materials and Methods). We sutured the right eyelid of mice from postnatal day (P) 21 to P4145 , an interval that encompassed the entire critical period for OD plasticity as defined by traditional single-unit recordings (Gordon and Stryker 1996). Visual evoked potentials (VEPs) in response to the presentation of horizontal bars (gratings) of different sizes (spatial frequencies) were then recorded. Gratings of lower spatial frequency evoked larger amplitudes (Guire et al. 1999; Lickey et al. 2004). In nondeprived mice, the contralateral eye always produced larger VEP amplitudes than did the ipsilateral eye, indicating the greater strength of the crossed pathway (Fig. 1A,B). As expected, deprivation of the right eye resulted in a decrease of the amplitude evoked by the right eye. Surprisingly, the amplitude evoked by the left (nondeprived) eye did not seem to increase (Fig. 1C,D)

A brief period of BE following LTMD from P21 to P41-44 partially eliminated the deprivation effect. The VEP amplitude evoked by the originally deprived right eye increased substantially following BE. Five or $10 \mathrm{~d}$ of BE produced a similar partial recovery of VEP amplitudes (Fig. 1E-H). Even after a longer period of LTMD from P21 to P56-60, $10 \mathrm{~d}$ of BE still produced partial recovery of deprived-eye responses (Fig. 1I,J). Responses evoked by the nondeprived (left) eye seemed unaffected by LTMD as well as subsequent BE.

To compare the effects of LTMD and BE, we averaged the amplitudes evoked by the two lowest spatial frequencies $(0.05$ and 0.065 cycles/degree, the two highest responses of the sweep). Because the three BE groups produced similar outcomes, we pooled these data into a single group and compared it with nondeprived and LTMD groups (Fig. 2A). A three-factor ANOVA (see Fig. 2A, legend) showed (1) that the right eye evoked smaller amplitude responses than the left eye, (2) that the uncrossed pathway evoked smaller amplitude responses than the crossed pathway, and (3) that there was a significant eye $\times$ experimental group interaction. The interaction indicated that LTMD and LTMD + BE affected right (deprived) eye responses more than left eye responses, which were essentially unchanged.

From the amplitude data given above, we computed an ocular dominance index (ODI) for each hemisphere. The ODI is a relative measure of the strength of the crossed (contralateral) divided by the crossed plus uncrossed (ipsilateral) responses. In nondeprived mice, ODIs of the right and left hemispheres were about equal (Fig. 2B). In contrast, deprivation of the right eye increased the ODI of the right hemisphere and decreased the ODI of the left hemisphere. This hemispheric difference in ODI was reduced after 5 or $10 \mathrm{~d}$ of $\mathrm{BE}$, reflecting the partial recovery of the right (deprived) eye responses. For statistics, we determined the right minus left hemispheric difference in ODI and also pooled the three BE groups (Fig. 2C). Compared with nondeprived mice, we found that LTMD caused a large and significant hemispheric difference; this difference was significantly reduced by BE (see Fig. 2C, legend).

\section{$B E$ and RS promotes nearly complete recovery from LTMD}

Opening the deprived right eye and closing the open left eye (RS) is thought to force the usage of the deprived eye, thereby increasing the maximal extent of recovery from MD (Dews and Wiesel 1970; Blakemore and Van Sluyters 1974). We therefore asked whether a period of RS, either by itself or following BE, could

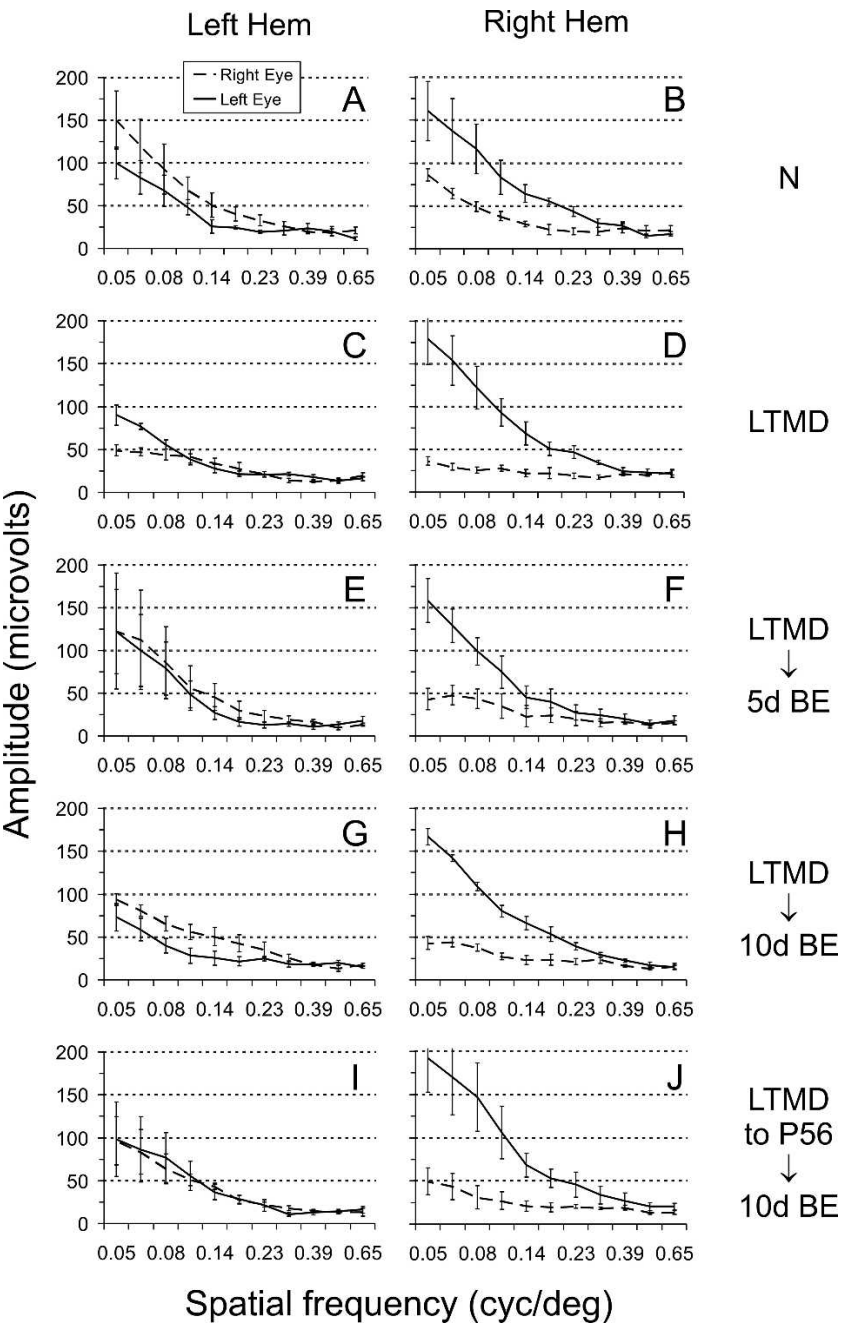

Figure 1. The amplitude of the VEP elicited from the deprived (right) eye decreases following LTMD and partially recovers following the restoration of $\mathrm{BE}$. The visual stimulus was a horizontal grating presented at a fixed contrast (88\%) but with variable spatial frequencies. The spatial frequencies used were (in cycles/degree) as follows: 0.05, 0.065, 0.084, $0.11,0.14,0.18,0.23,0.3,0.39,0.5,0.65$. Curves depict mean VEP amplitudes in microvolts as a function of spatial frequency. $(A, B)$ Mean responses from the left and right hemispheres of four nondeprived $(N)$ P41-47 mice. $(C, D)$ Mean responses from five mice immediately after right eye LTMD from P21 to P41-45. ( $E, F)$ Mean responses from five mice after right eye LTMD from P21 to P41-45 plus 5 d of BE. $(G, H)$ Mean responses from five mice after right eye LTMD from P21 to P41-45 plus $10 \mathrm{~d}$ of BE. $(I, /)$ Mean responses from four mice after LTMD of the right eye from P21 to P56-60 plus $10 \mathrm{~d}$ of BE. Left Hem indicates left hemisphere; right Hem, right hemisphere. Error bars, SEM.

facilitate recovery. We deprived the right eye of mice from P14-21 to either P61-65 (young adults) or P92-158 (mature adults), at which time we either recorded from the animals or subjected them to one of two recovery protocols: (1) opening the right eye for 6-7 d and then suturing shut the left eye for $4 \mathrm{~d}$ (denoted as BE+RS), or (2) reverse suturing for 4-7 d (RS) without prior BE.

As before, LTMD dramatically suppressed the amplitude of cortical potentials evoked by the right eye compared with the left eye (Fig. 3A,B). Note in particular that the response of the right eye in the right hemisphere (right eye uncrossed pathway) was nearly undetectable (Fig. 3B). Surprisingly, this effect of LTMD appeared to be nearly completely reversed by BE+RS. In both 
A

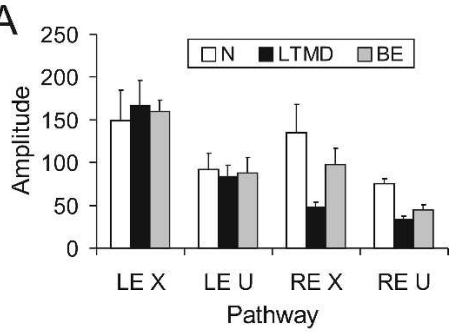

B
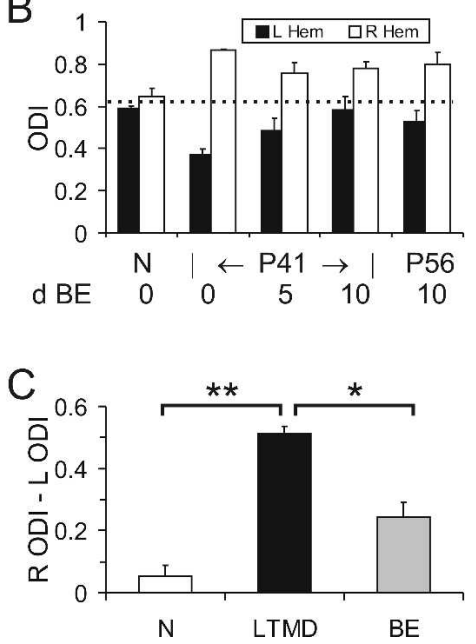

Figure 2. Both VEP amplitudes and ocular dominance indexes (ODIs) show evidence of partial recovery following $B E$. The data presented here are from the same mice as in Figure 1. (A) Average of amplitudes (in microvolts) evoked by the two lowest spatial frequencies, 0.065 and 0.05 cycles/degree, presented at $88 \%$ contrast for each pathway of each eye. Three-factor ANOVA showed an effect of eye $(P<0.0001)$, pathway $(P<0.0001)$, and an eye $\times$ experimental group interaction $(P=0.04) . \mathrm{N}$ indicates nondeprived (four mice); LTMD, right eye deprived (five mice); $B E$, LTMD followed by 5-10 d of BE (13 mice, all LTMD + BE groups pooled). LE X indicates left eye crossed pathway; LE U, left eye uncrossed pathway; RE X, right eye crossed pathway; and RE U, right eye uncrossed pathway. (B) ODI values for each hemisphere of each experimental group. N indicates nondeprived controls; P41 or P56, deprivation from P21 to P41-45 or P56-60. d BE denotes the number of days of BE. The dotted line represents the mean of right and left hemisphere ODIs for nondeprived mice. (C) ODI of the right hemisphere minus ODI of the left hemisphere for nondeprived ( $N$, four mice), right-eye deprived (LTMD, five mice), and LTMD followed by 5-10 d BE (BE, 13 mice). There was a significant difference between the three groups overall (one-factor ANOVA, $P=0.001)$. ${ }^{* \star} P=0.003$, Bonferroni post-hoc test; ${ }^{*} P=0.03$, Bonferroni post-hoc test.

young adults (four mice) and mature adults (five mice), visual cortical responses indistinguishable from normal were observed, and therefore, the results were pooled (Fig. 3C,D). Note that responses of the right eye were roughly equal to that of the left eye (Fig. 3C,D). The dramatic recovery produced by BE $+\mathrm{RS}$ led us to ask whether RS alone might induce significant recovery. Indeed, a short period of RS, 4-7 d, restored right eye amplitudes to nearnormal levels (Fig. 3, cf. A,B and E,F).

For statistics, we averaged the amplitudes for the two lowest spatial frequencies and analyzed significance by using threefactor ANOVAs (for statistics, see Fig. 4, legends). Here, we analyzed responses elicited by both $88 \%$ and $44 \%$ contrast gratings. Restoration of amplitudes was evident at both contrast levels; however, recovery appeared more complete and significant at $88 \%$ contrast. Visual recovery induced by BE + RS or RS alone was mainly a consequence of increased right eye amplitudes; left eye amplitudes showed little consistent change (Fig. 4A,B). The eye- specific effect of experimental treatments was confirmed by a significant eye $\times$ treatment group interaction for data collected at $88 \%$ contrast (see Fig. 4 , legend).

ODI values provide further evidence for recovery from LTMD (Fig. 4C,D). At both $88 \%$ and $44 \%$ contrast, the left/right hemispheric difference produced by LTMD disappeared following BE + RS. Even with RS alone, nearly complete recovery was observed at $88 \%$ contrast. The left/right difference in ODIs was not significant in the RS cases at $88 \%$ contrast; however, at $44 \%$ contrast, the left/right difference persisted. To compare the magnitude of the hemispheric difference between groups, we computed R-ODI minus L-ODI values for all cases. We found statistically significant reductions in the value of the left/right ODI difference between LTMD and recovery groups for both $88 \%$ and $44 \%$ contrast (Fig. 4E,F).

VEP thresholds are known to correlate with psychophysical thresholds (Tyler et al. 1979; Allen et al. 1986). Therefore, to assess whether the observed recovery of OD and VEP amplitudes translates into improved visual acuity, we determined the threshold of VEP detection for each eye. The threshold was defined as the lowest spatial frequency that failed to elicit a VEP response above the level of noise (see Materials and Methods). After LTMD, right eye thresholds were reduced compared with those of the left eye, particularly in the uncrossed pathway (Fig. $5 \mathrm{~A}, \mathrm{~B})$. Both recovery paradigms, $\mathrm{BE}+\mathrm{RS}$ and RS alone, eliminated the difference in acuity between the right and left eyes largely by enhancing the acuity of the right eye (Fig. 5C-F). Note that the recovery observed with $88 \%$ contrast gratings was very similar to that observed at $44 \%$ contrast (Fig. 5, cf. A,C,E and $\mathrm{B}, \mathrm{D}, \mathrm{F})$.

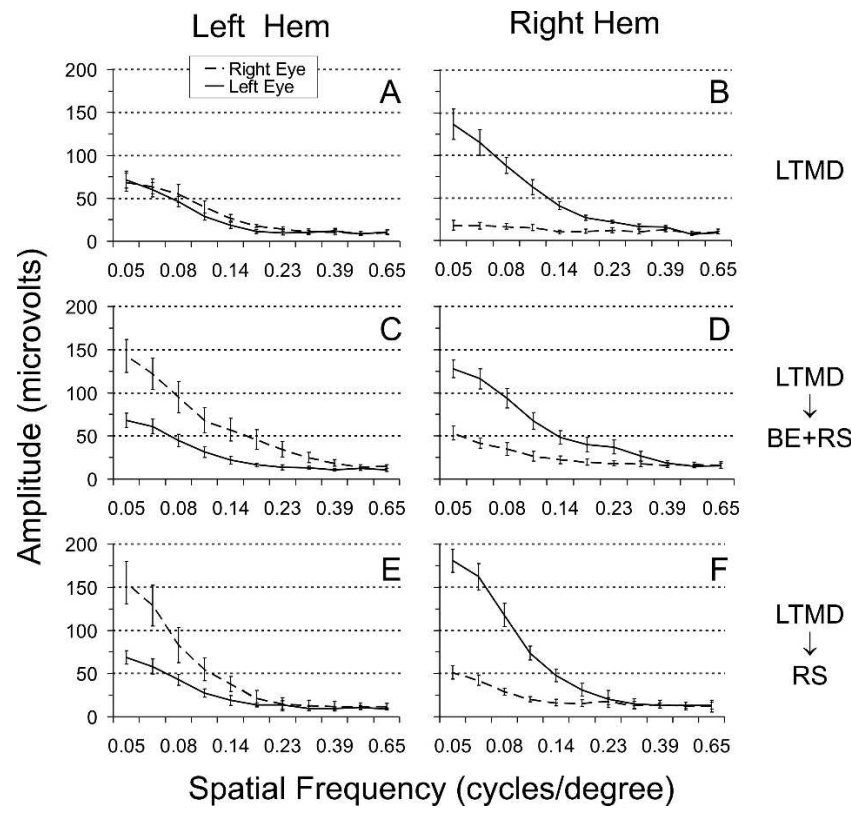

Figure 3. VEP responses strongly shift toward the left eye following right eye LTMD but substantially recovered following BE + RS or RS alone. The visual stimulus was a horizontal grating presented at a fixed contrast (88\%) but with variable spatial frequency (for details, see Fig. 1). (A,B) Mean VEP amplitudes, shown as a function of spatial frequency of the stimulus, for the left $(A)$ and right $(B)$ hemispheres of five mice with right eye LTMD from P21 to P62-115 (LTMD). (C,D) Mean VEP amplitudes for the left $(C)$ and right $(D)$ hemispheres of nine mice with right eye LTMD from P14-21 to P61-102 followed by 6-7 d of BE and $4 \mathrm{~d}$ of RS $(\mathrm{LTMD} \rightarrow \mathrm{BE}+\mathrm{RS})$. $(E, F)$ Mean VEP amplitudes for the left $(E)$ and right $(F)$ hemispheres of five mice after right eye LTMD from P14-21 to P97-158 followed by $4-7 \mathrm{~d}$ of RS (LTMD $\rightarrow$ RS). 

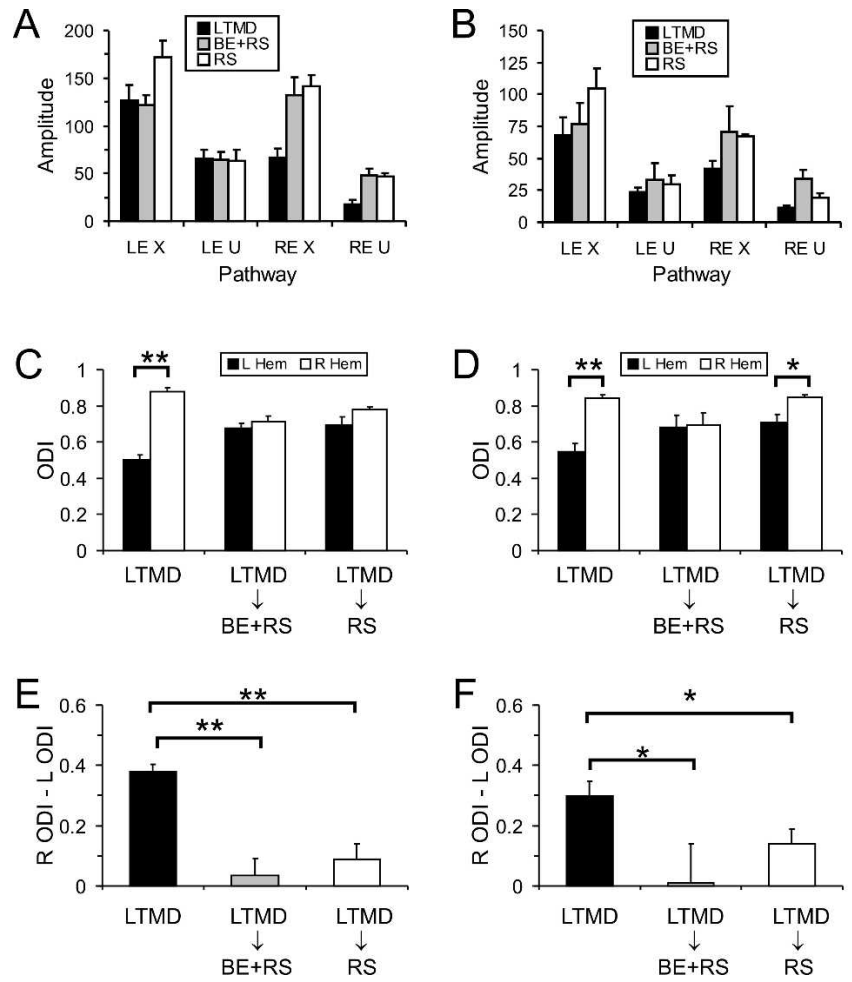

Figure 4. RS efficiently promoted recovery of VEP amplitudes and ODI values following LTMD. The data presented here are from the same mice as in Figure 3. Visual stimuli were presented at $88 \%$ contrast $(A, C, E)$, or $44 \%$ contrast $(B, D, F) .(A, B)$ Average of amplitudes (in microvolts) evoked by the two highest spatial frequency stimuli for each pathway of each eye in mice subjected to LTMD (LTMD; $N=5$ for panel $A, 4$ for panel $B$ ), LTMD followed by $B E+R S(B E+R S ; N=9,5)$, and LTMD followed by RS mice (RS; $N=5,5$ ). Separate three-factor ANOVAs calculated for data collected at $88 \%$ or $44 \%$ contrast showed significant effects of eye ( $P=0.0009$ for $88 \%, P=0.02$ for $44 \%$ ) and pathway (crossed or uncrossed; $P<0.0001$ for $88 \%, P<0.0001$ for $44 \%$ ). There was a significant eye $\times$ treatment group interaction for stimulation at $88 \%$ contrast $(P=0.0165)$ but not at $44 \%$ contrast $(P=0.31) .(C, D)$ ODI values for each hemisphere of mice from the same groups as in $A$ and $B$. Only the LTMD group showed a significant difference between left and right hemisphere ODI for stimulation at $88 \%$ contrast (C), while both LTMD and LTMD $\rightarrow$ RS groups were significantly different for stimulation at $44 \%$ contrast $(D) .{ }^{*} P=0.02$ (paired $t$-test); ${ }^{* *} P \leq 0.0001$ (paired $t$-test). $(E, F$ The hemispheric difference in ODI caused by LTMD was almost completely eliminated following BE + RS or RS for responses elicited at $88 \%$ contrast $(E)$, whereas recovery was more variable but still significant at $44 \%$ contrast $(F) .{ }^{*} P<0.05, t$-test; ${ }^{*} P<0.002, t$-test.

\section{Visual recovery in adulthood can be detected by single unit recording}

Finally, we considered the possibility that the dramatic recovery that we have found resulted from complex multicellular interactions revealed by population indexes of activity, but might not be detected at the level of single cells. To investigate this issue, we recorded single-unit responses from a subset of the mice in which we had recorded VEPs. The OD of single units sampled through the entire depth of binocular V1 was scored by an observer blind to treatment condition using the seven-point scale of Hubel and Wiesel (1962). Whereas the single-unit OD histogram is contralaterally biased in normal mice (Fig. 6A), single units recorded from the left binocular V1 of LTMD mice were shifted toward the ipsilateral open eye (Fig. 6B). In contrast, mice that received LTMD followed by $\mathrm{BE}+\mathrm{RS}$ displayed a contralaterally biased OD distribution closely resembling normal mice (Fig. 6; cf. $\mathrm{C}$ and $\mathrm{A}$ ). Contralateral bias indexes (CBI, see Materials and
Methods) derived from OD scores of single units demonstrate that $\mathrm{BE}+\mathrm{RS}$ completely normalized the OD of single units (Fig. $6 \mathrm{D})$.

\section{Discussion}

There are now clear examples in several model systems supporting the assertion that cortical maps can be modified in adulthood as well as in development, evidence of the remarkable adaptability of the adult brain. However, it is not clear whether adult and juvenile experiences alter the cortex in similar and perhaps interchangeable ways. In this article, we have asked whether the abnormal function of circuits resulting from long-term visual deprivation starting in development can be ameliorated by adult experience well past the critical period. Unexpectedly, we found that in mice the detrimental effects of LTMD can be nearly completely reversed by a regimen of binocular vision and reverse eye closure. Thus, our data suggest that even in V1 adult visual experience can promote development of visual functions that have failed to develop properly as a result of deprivation early in life.

Our findings are consistent with, and provide a neural basis for, observations of recovery of visual acuity following LTMD in rodents, cats, monkeys, and humans. In humans, a recent multicenter clinical trial found that adolescents near or past puberty (13-17 yr old) who were previously not treated for amblyopia can benefit from a regimen of reverse occlusion (Scheiman et al. 2005). Additionally, smaller case studies have long suggested that adult amblyopic patients can improve their acuity by visual training (Levi and Polat 1996; Polat et al. 2004; Levi 2005) or occlusion therapy (Selenow and Ciuffreda 1986; Saulles 1987; Rutstein and Fuhr 1992; Wick et al. 1992; Park et al. 2004). In cats, behavioral studies by Mitchell (1988) documented significant, though incomplete, recovery of vision in adulthood following deprivation from eye opening. In cats, monkeys, and humans, vision in the amblyopic eye frequently improves following enucleation of the normal eye (Smith 1981; Smith et al. 1982; Harwerth et al. 1986; El Mallah et al. 2000).

Yet several previous electrophysiological studies of cats found that recovery from MD starting near birth can be elicited only within the first several months of life. Wiesel and Hubel (1965) found that an MD from birth to 3 mo of age cannot be reversed to a significant extent. They reported that even RS for 1 yr produced little recovery of OD. Several subsequent studies confirmed these findings (Hubel and Wiesel 1970; Blakemore and Van Sluyters 1974; Movshon 1976), collectively suggesting that in cats the potential for recovery declines and disappears over the first $4 \mathrm{mo}$ of life. The startling dissociation between behavioral and electrophysiological outcomes was even noted in a single case that was studied by both electrophysiology and behavior; there was behavioral recovery but no recovery detectable by single unit recordings (Dews and Wiesel 1970).

There are three possible explanations for the discrepancy between electrophysiology and behavior. First, behavioral tests might detect recovery that occurs at cortical levels beyond V1. Second, previous single unit studies of V1 may not have equally sampled all cortical layers and/or columns and hence might not adequately represent the cells that recover. Third, animals might need to be engaged in ethologically relevant visual tasks in order to improve their acuity. The first explanation seems improbable, since it posits that near-normal acuity can be achieved in the absence of significant recovery detectable in V1, the site of processing of simple visual forms. The second explanation is clearly possible, especially in light of our current data showing substantial recovery of adult V1. Recent findings have shown that the detection of adult OD plasticity can be masked by anesthetics that enhance $\mathrm{GABA}_{\mathrm{A}}$ receptors (Pham et al. 2004; Heimel et al. 

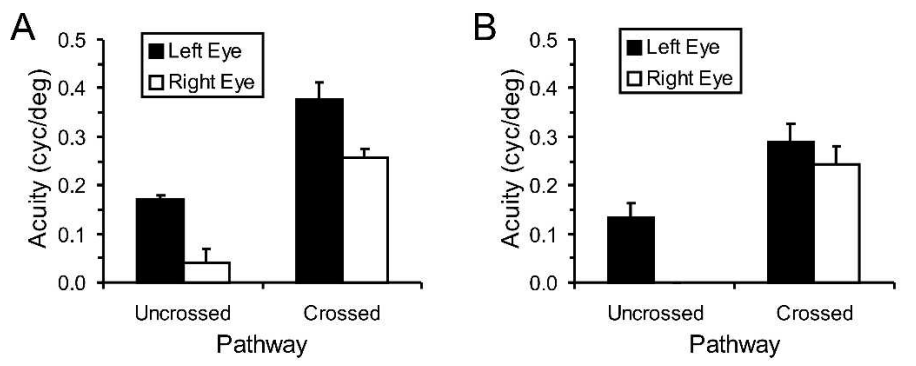

LTMD
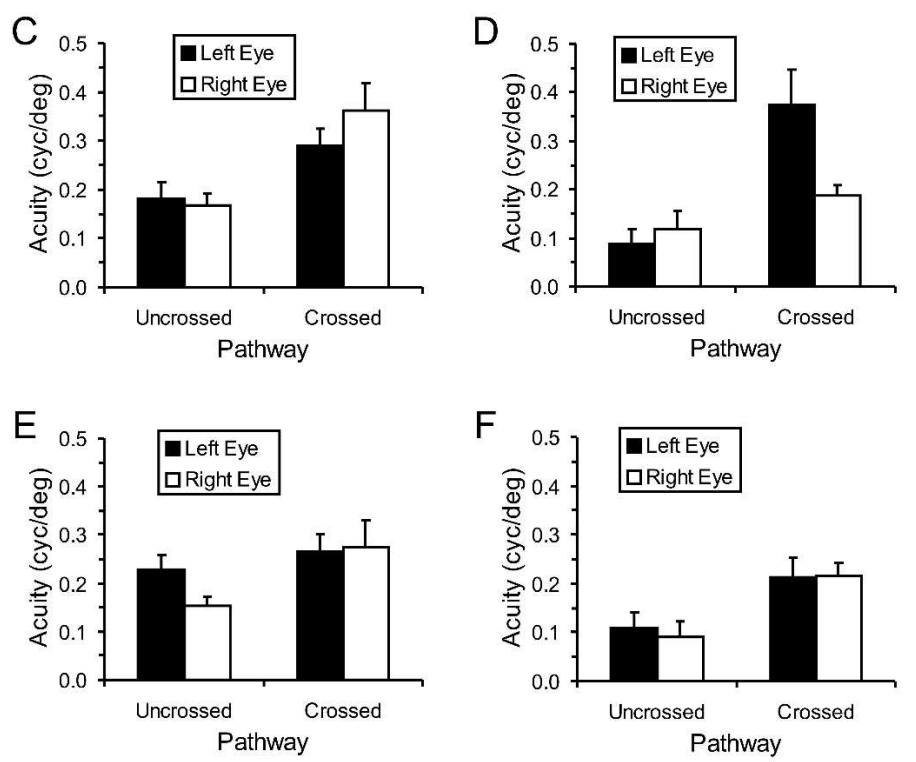

Figure 5. Acuity of the deprived eye is severely impaired by LTMD but substantially recovers following $B E+R S$ or RS. The data presented here are from the same mice as in Figures 3 and 4 . VEPs were elicited by gratings of $88 \%$ contrast $(A, C, E)$ or $44 \%$ contrast $(B, D, F)$. Spatial frequency thresholds, a correlate of behavioral acuity, are shown for the uncrossed and crossed pathways of each eye in mice with the following: $(A, B) \operatorname{LTMD}(N=5$ mice for panel $A, 4$ mice for panel $B),(C, D)$ LTMD followed by $\mathrm{BE}+\mathrm{RS}(N=9,5)$, or $(E, F)$ LTMD followed by RS $(N=5,5)$. Separate three-factor ANOVAs calculated for data obtained with $88 \%$ or $44 \%$ contrast stimuli. Statistics showed significant effects of eye $(P=0.04$ for $88 \%$ contrast, $P=0.02$ for $44 \%$ contrast $)$ and pathway $(P<0.0001$ for $88 \%$ contrast, $P<0.0001$ for $44 \%$ contrast). There was a significant eye $\times$ treatment group interaction for acuity at $88 \%$ contrast $(P<0.02)$; there was a significant eye $\times$ pathway $\times$ treatment group interaction at $44 \%$ contrast $(P=0.0079)$. The significant eye $\times$ group interaction for stimulation at $88 \%$ contrast suggests that both recovery regimens (BE+RS or RS) improved the acuity of the right (formerly deprived) eye. The significant eye $\times$ pathway $\times$ treatment group interaction at $44 \%$ contrast suggests a greater effect in one pathway; it is apparent from the data that the uncrossed pathway is affected more than the crossed by LTMD and recovery regimens.

2007). Here we have recorded single units throughout the cortical thickness under urethane anesthesia and have found nearly complete recovery. Finally, the third explanation suggests that animals might recover best when they are actively engaged in visual tasks, as they normally would do if they were in a natural environment (Sale et al. 2007). In this regard, repeated visual testing by using behavioral measures might act as a form of visual training.

We found that RS was much more effective than BE in promoting visual recovery. In agreement with our findings, Iny et al. (2006) found that BE produced partial recovery of behavioral acuity in adult rats. However, Pizzorusso et al. (2006) reported little recovery in adult rats following $1 \mathrm{wk}$ of RS. We cannot explain why their results differ so dramatically from ours, but it is notable that they also did not observe adult OD plasticity in mice with VEP (Huang et al. 1999). Perhaps there are unknown but critical differences in methods of recording. Our usage of BE plus RS might be the most effective recovery paradigm because although BE stimulates a faster onset of visual recovery, RS allows a greater extent of functional gain (Mitchell et al. 1977, 2001; Giffin and Mitchell 1978). In the long-term, RS produces a greater extent of recovery because it eventually shifts competitive mechanisms in favor of the initially deprived eye (Mitchell et al. 2001).

Whereas visual recovery enhanced the magnitude of field potentials evoked by deprived eye inputs, LTMD itself only caused depression of VEPs elicited from the deprived eye. These observations suggest that the mechanisms underlying LTMD are not the same as those that mediate recovery. Several lines of evidence support the hypothesis that distinct mechanisms mediate the effect of MD compared with recovery. First, the effect of MD requires protein synthesis and CREB activity (Taha and Stryker 2002; Mower et al. 2002), whereas recovery does not (Krahe et al. 2005). Second, sleep is required for the effect of MD but not for recovery (Frank et al. 2001; Dadvand et al. 2006). Third, the immediateearly gene tissue plasminogen activator is required for the effect of recovery but not for the preceding MD (Muller and Griesinger 1998).

It is surprising that LTMD did not cause potentiation of nondeprived inputs, as was reported for short-term MD of $5 \mathrm{~d}$ to $2 \mathrm{wk}$ (Frenkel and Bear 2004). Prior studies of LTMD were uninformative on this issue because they used relative measures of the size and/or strength of eye-specific pathways. For example, an assay of OD based on unit recordings is a relative measure of the response to the deprived eye versus the nondeprived eye. It is possible that potentiation of the nondeprived pathway in short-term MD is transient. Long-term enhancement of open eye pathways might be mediated by an expansion of the cortical area influenced by the open eye (Shatz and Stryker 1978; Antonini and Stryker 1996; Antonini et al. 1999), rather than by changes in the density of electrical activity generated.

Mice are ubiquitously used to model human disease, but we cannot exclude the possibility that our results in mice may not be entirely applicable to humans and other mammalian species. Recovery in humans would not be as rapid since development of mice is accelerated compared with that of humans. The lifespan of mice is 30-fold shorter than that of humans, and its heart rate 10 times faster. However, despite differences in metabolic and maturational rates, the basic molecular machinery, cellular types, and visual circuitry are conserved, suggesting that similar mechanisms are likely to operate in mice and humans. Our finding of dramatic recovery of vision in mice following LTMD poses several important questions. Is the recovery maintained stably over time? What are the mechanisms that mediate recovery and can they be enhanced? Answers to these questions may yield insights into the treatment of adult humans with amblyopia. 


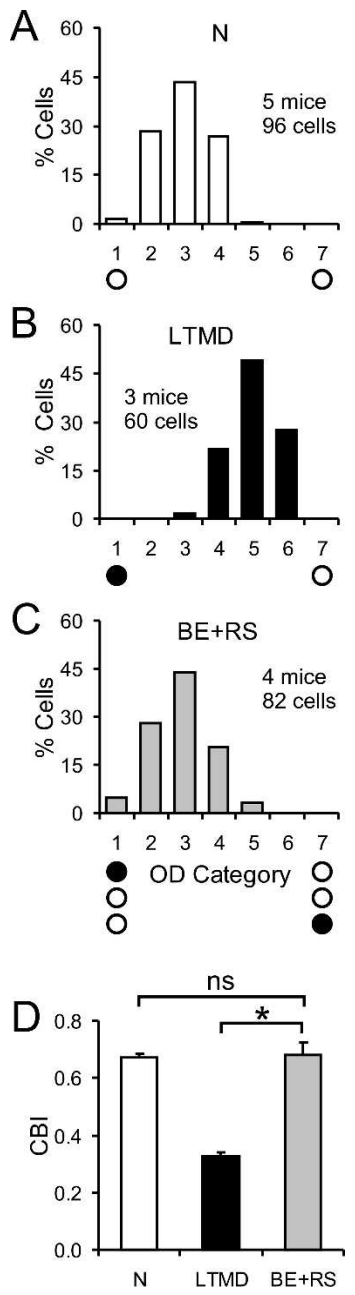

Figure 6. $A B E+R S$ regimen promotes recovery of single units from LTMD. OD was scored by an observer blind to treatment condition using the classical seven-point scale ( 1 , only stimulated by the contralateral eye; 4 , equally stimulated by both eyes; 7 , only stimulated by the ipsilateral eye). Open circles represent the open eye; filled circles represent the deprived eye. (A) OD histograms of single-unit responses in nondeprived mice aged P55-110. Note that these data were taken from Fischer et al. (2007) and are shown here only for comparison. (B) OD histograms after LTMD from P21 to P104-113. (C) OD histograms after LTMD from P21 to P92-95 followed by $6 \mathrm{~d}$ of BE plus $4 \mathrm{~d}$ of RS. (D) Contralateral bias indexes ( $\mathrm{CBI}$; see Materials and Methods) derived from individual animal histograms. A one-factor ANOVA showed a significant effect of treatment group $(P<0.0001)$. ns, $P=0.86$, Bonferroni post-hoc test; ${ }^{*} P<0.0001$, Bonferroni post-hoc test. Conventions as in Figures 4 and 5 .

\section{Materials and Methods}

\section{Housing and care of mice}

All procedures used in this study were approved by the Institutional Animal Care and Use Committee of Baylor College of Medicine and conform to the guidelines of the National Institutes of Health. We used C57BL/6 mice purchased from either the Jackson Laboratory (Bar Harbor, ME) or Taconic Farms Inc. or bred from these stocks. Mice were housed under a standard 12-h light/12-h dark schedule in standard transparent plastic cages (26 $\mathrm{cm} \times 15 \mathrm{~cm} \times 13 \mathrm{~cm}$ ). Food and water was provided ad lib. They were usually kept two to four mice per cage.

\section{Deprivation and recovery surgeries}

MD was performed under isoflurane anesthesia (Attane 1\%-2\%, Minrad), as described previously (Lickey et al. 2004; Pham et al.
2004; Fischer et al. 2007). Following opening of the deprived eye, a sterile ophthalmic lubricating ointment was applied daily to keep the eye from drying out. Animals were checked daily during the recovery period.

\section{VEP recordings}

VEP recordings were made under urethane anesthesia $(1.5 \mathrm{mg} / \mathrm{g}$ body weight, i.p.) using standard methods (Lickey et al. 2004; Pham et al. 2004; Fischer et al. 2007). Briefly, PowerDiva software from Anthony Norcia (Smith Kettlewell Institute of Visual Sciences) was used for data acquisition and analysis. The visual stimulus was a horizontal sinusoidal grating covering the central $80^{\circ}$ of the visual field $\left(40^{\circ}\right.$ on either side of the midline). The grating reversed phase about four times per sec $(2.02 \mathrm{~Hz})$. One stimulus presentation (one trial) consisted of a spatial frequency sweep increasing from 0.65 to 0.05 cycles/degree in 10 log steps. Stimuli were presented at $44 \%$ and $88 \%$ contrasts. Trials alternated between right and left eyes until 16 trials were completed.

\section{Analysis of VEP data}

Analyses of the raw data were performed using PowerDiva software, as described previously (Lickey et al. 2004; Pham et al. 2004; Fischer et al. 2007). Spatial frequency thresholds were derived using the algorithm basically as described by Fischer et al. (2007). The data of one spatial frequency sweep fell into 11 bins of decreasing spatial frequency. The spatial frequency bins used were (in cycles/degree): $0.65,0.5,0.39,0.3,0.23,0.18,0.14,0.11$, $0.084,0.065$, and 0.05 . The mean and standard deviation of noise was calculated across the 11 bins. Threshold was defined as being equal to the bin value preceding the bin where the signal exceeded the noise plus one standard deviation of the noise. If the signal always exceeded this value, the threshold was assigned the value of 0.65 . If the signal never exceeded this value, the threshold was assigned the value of 0.0 cycles/degree.

For each hemisphere of each mouse or group of mice, we also calculated an ODI according to the formula ODI $=\mathrm{X} /$ $(X+U)$, where $X=$ the VEP amplitude of the crossed pathway and $\mathrm{U}=$ the VEP amplitude of the uncrossed pathway. ODIs were calculated using the mean of the data obtained at the two lowest spatial frequencies (i.e., 0.065 and 0.05 cycles/degree).

\section{Single-unit recordings}

Single-unit recordings were made using standard procedures (Fischer et al. 2004, 2007). Briefly, animals were anesthetized with urethane $(1.5 \mathrm{mg} / \mathrm{g}$ body weight, i.p.), and units were sampled throughout the cortical thickness in three to four penetrations evenly spaced across the mediolateral extent of binocular V1 in the left hemisphere. The OD of units was scored on a one-to-seven scale by an observer blind to treatment condition (nondeprived, LTMD, LTMD with BE + RS) of the animals. For each animal or group of animals, we also calculated a CBI using the formula CBI $=\left[\left(n_{1}-n_{7}\right)+(2 / 3)\left(n_{2}-n_{6}\right)+(1 / 3)\right.$ $\left.\left(n_{3}-n_{5}\right)+N\right] / 2 N$, where $N$ is the total number of cells and $n_{\mathrm{i}}$ is the number of cells in OD category $i$.

\section{Acknowledgments}

We thank Marvin Lickey, Nigel Daw, Elizabeth Quinlan, and Ronald Davis for helpful discussions and/or review of the manuscript, and Jim Legare and Marium Waqar for technical assistance. This research was supported by National Eye Institute grant R01 EY014238, National Institute of Mental Health grant K08 MH01971, and a start-up grant from Baylor College of Medicine to T.A.P.

\section{References}

Allard, T., Clark, S.A., Jenkins, W.M., and Merzenich, M.M. 1991 Reorganization of somatosensory area $3 \mathrm{~b}$ representations in adult owl monkeys after digital syndactyly. J. Neurophysiol. 66: 1048-1058.

Allen, D., Norcia, A.M., and Tyler, C.W. 1986. Comparative study of electrophysiological and psychophysical measurement of the contrast sensitivity function in humans. Am. J. Optom. Physiol. Opt. 
63: $442-449$

Antonini, A. and Stryker, M.P. 1996. Plasticity of geniculocortical afferents following brief or prolonged monocular occlusion in the cat. J. Comp. Neurol. 369: 64-82.

Antonini, A., Fagiolini, M., and Stryker, M.P. 1999. Anatomical correlates of functional plasticity in mouse visual cortex. J. Neurosci. 19: $4388-4406$.

Blakemore, C. and Van Sluyters, R.C. 1974. Reversal of the physiological effects of monocular deprivation in kittens: Further evidence for a sensitive period. J. Physiol. 237: 195-216.

Blakemore, C., Garey, L.J., and Vital-Durand, F. 1978. The physiological effects of monocular deprivation and their reversal in the monkey's visual cortex. J. Physiol. 283: 223-262.

Blakemore, C., Vital-Durand, F., and Garey, L.J. 1981. Recovery from monocular deprivation in the monkey. I. Reversal of physiological effects in the visual cortex. Proc. R. Soc. Lond. B. Biol. Sci. 213: 399-423.

Dadvand, L., Stryker, M.P., and Frank, M.G. 2006. Sleep does not enhance the recovery of deprived eye responses in developing visual cortex. Neuroscience 143: 815-826.

Dews, P.B. and Wiesel, T.N. 1970. Consequences of monocular deprivation on visual behaviour in kittens. J. Physiol. 206: 437-455

El Mallah, M.K., Chakravarthy, U., and Hart, P.M. 2000. Amblyopia: Is visual loss permanent? Br. J. Ophthalmol. 84: 952-956.

Fagiolini, M., Pizzorusso, T., Berardi, N., Domenici, L., and Maffei, L. 1994. Functional postnatal development of the rat primary visual cortex and the role of visual experience: Dark rearing and monocular deprivation. Vision Res. 34: 709-720.

Fischer, Q.S., Beaver, C.J., Yang, Y., Rao, Y., Jakobsdottir, K.B., Storm, D.R., McKnight, G.S., and Daw, N.W. 2004. Requirement for the RII $\beta$ isoform of PKA, but not calcium-stimulated adenylyl cyclase, in visual cortical plasticity. J. Neurosci. 24: 9049-9058.

Fischer, Q.S., Graves, A., Evans, S., Lickey, M.E., and Pham, T.A. 2007. Monocular deprivation in adult mice alters visual acuity and single-unit activity. Learn. Mem. 14: 277-286.

Fox, K. 1992. A critical period for experience-dependent synaptic plasticity in rat barrel cortex. J. Neurosci. 12: 1826-1838.

Fox, K. 1994. The cortical component of experience-dependent synaptic plasticity in the rat barrel cortex. J. Neurosci. 14: 7665-7679.

Frank, M.G., Issa, N.P., and Stryker, M.P. 2001. Sleep enhances plasticity in the developing visual cortex. Neuron 30: 275-287.

Freeman, R.D. and Olson, C. 1982. Brief periods of monocular deprivation in kittens: Effects of delay prior to physiological study. J. Neurophysiol. 47: 139-150.

Frenkel, M.Y. and Bear, M.F. 2004. How monocular deprivation shifts ocular dominance in visual cortex of young mice. Neuron 44: $917-923$

Giffin, F. and Mitchell, D.E. 1978. The rate of recovery of vision after early monocular deprivation in kittens. J. Physiol. 274: 511-537.

Gordon, J.A. and Stryker, M.P. 1996. Experience-dependent plasticity of binocular responses in the primary visual cortex of the mouse. $J$. Neurosci. 16: 3274-3286.

Guire, E.S., Lickey, M.E., and Gordon, B. 1999. Critical period for the monocular deprivation effect in rats: Assessment with sweep visually evoked potentials. J. Neurophysiol. 81: 121-128.

Harwerth, R.S., Smith, E.L., Duncan, G.C., Crawford, M.L., and von Noorden, G.K. 1986. Effects of enucleation of the fixating eye on strabismic amblyopia in monkeys. Invest. Ophthalmol. Vis. Sci. 27: 246-254.

Heimel, J.A., Hartman, R.J., Hermans, J.M., and Levelt, C.N. 2007. Screening mouse vision with intrinsic signal optical imaging. Eur. J. Neurosci. 25: 795-804

Hofer, S.B., Mrsic-Flogel, T.D., Bonhoeffer, T., and Hubener, M. 2006. Prior experience enhances plasticity in adult visual cortex. Nat. Neurosci. 9: 127-132.

Huang, Z.J., Kirkwood, A., Pizzorusso, T., Porciatti, V., Morales, B., Bear, M.F., Maffei, L., and Tonegawa, S. 1999. BDNF regulates the maturation of inhibition and the critical period of plasticity in mouse visual cortex. Cell 98: 739-755.

Hubel, D.H. and Wiesel, T.N. 1962. Receptive fields, binocular interaction and functional architecture in the cat's visual cortex. J. Physiol. 160: 106-154.

Hubel, D.H. and Wiesel, T.N. 1970. The period of susceptibility to the physiological effects of unilateral eye closure in kittens. J. Physiol. 206: $419-436$.

Iny, K., Heynen, A.J., Sklar, E., and Bear, M.F. 2006. Bidirectional modifications of visual acuity induced by monocular deprivation in juvenile and adult rats. J. Neurosci. 26: 7368-7374.

Jenkins, W.M., Merzenich, M.M., Ochs, M.T., Allard, T., and Guic-Robles, E. 1990. Functional reorganization of primary somatosensory cortex in adult owl monkeys after behaviorally controlled tactile stimulation. J. Neurophysiol. 63: 82-104.
Kaas, J.H. and Qi, H.X. 2004. The reorganization of the motor system in primates after the loss of a limb. Restor. Neurol. Neurosci. 22: $145-152$.

Kind, P.C., Mitchell, D.E., Ahmed, B., Blakemore, C., Bonhoeffer, T., and Sengpiel, F. 2002. Correlated binocular activity guides recovery from monocular deprivation. Nature 416: 430-433.

Krahe, T.E., Medina, A.E., Bittencourt-Navarrete, R.E., Colello, R.J., and Ramoa, A.S. 2005. Protein synthesis-independent plasticity mediates rapid and precise recovery of deprived eye responses. Neuron 48: $329-343$.

Levi, D.M. 2005. Perceptual learning in adults with amblyopia: A reevaluation of critical periods in human vision. Dev. Psychobiol. 46: 222-232.

Levi, D.M. and Polat, U. 1996. Neural plasticity in adults with amblyopia. Proc. Natl. Acad. Sci. 93: 6830-6834.

Lickey, M.E., Pham, T.A., and Gordon, B. 2004. Swept contrast visual evoked potentials and their plasticity following monocular deprivation in mice. Vision Res. 44: 3381-3387.

Merzenich, M.M., Nelson, R.J., Stryker, M.P., Cynader, M.S., Schoppmann, A., and Zook, J.M. 1984. Somatosensory cortical map changes following digit amputation in adult monkeys. J. Comp. Neurol. 224: 591-605.

Mitchell, D.E. 1988. The extent of visual recovery from early monocular or binocular visual deprivation in kittens. J. Physiol. 395: 639-660.

Mitchell, D.E., Cynader, M., and Movshon, J.A. 1977. Recovery from the effects of monocular deprivation in kittens. J. Comp. Neurol. 176: $53-63$.

Mitchell, D.E., Gingras, G., and Kind, P.C. 2001. Initial recovery of vision after early monocular deprivation in kittens is faster when both eyes are open. Proc. Natl. Acad. Sci. 98: 11662-11667.

Movshon, J.A. 1976. Reversal of the physiological effects of monocular deprivation in the kitten's visual cortex. J. Physiol. 261: 125-174.

Mower, A.F., Liao, D.S., Nestler, E.J., Neve, R.L., and Ramoa, A.S. 2002. $\mathrm{cAMP} / \mathrm{Ca}^{2+}$ response element-binding protein function is essential for ocular dominance plasticity. J. Neurosci. 22: 2237-2245.

Muller, C.M. and Griesinger, C.B. 1998. Tissue plasminogen activator mediates reverse occlusion plasticity in visual cortex. Nat. Neurosci. 1: $47-53$.

Olson, C.R. and Freeman, R.D. 1975. Progressive changes in kitten striate cortex during monocular vision. J. Neurophysiol. 38: 26-32.

Park, K.H., Hwang, J.M., and Ahn, J.K. 2004. Efficacy of amblyopia therapy initiated after 9 years of age. Eye 18: 571-574

Pham, T.A., Graham, S.J., Suzuki, S., Barco, A., Kandel, E.R., Gordon, B., and Lickey, M.E. 2004. A semi-persistent adult ocular dominance plasticity in visual cortex is stabilized by activated CREB. Learn. Mem. 11: 738-747.

Pizzorusso, T., Medini, P., Landi, S., Baldini, S., Berardi, N., and Maffei, L. 2006. Structural and functional recovery from early monocular deprivation in adult rats. Proc. Natl. Acad. Sci. 103: 8517-8522.

Polat, U., Ma-Naim, T., Belkin, M., and Sagi, D. 2004. Improving vision in adult amblyopia by perceptual learning. Proc. Natl. Acad. Sci. 101: 6692-6697.

Qi, H.X., Stepniewska, I., and Kaas, J.H. 2000. Reorganization of primary motor cortex in adult macaque monkeys with long-standing amputations. J. Neurophysiol. 84: 2133-2147.

Rutstein, R.P. and Fuhr, P.S. 1992. Efficacy and stability of amblyopia therapy. Optom. Vis. Sci. 69: 747-754.

Sale, A., Maya Vetencourt, J.F., Medini, P., Cenni, M.C., Baroncelli, L., De Pasquale, R., and Maffei, L. 2007. Environmental enrichment in adulthood promotes amblyopia recovery through a reduction of intracortical inhibition. Nat. Neurosci. 10: 679-681.

Saulles, H. 1987. Treatment of refractive amblyopia in adults. J. Am. Optom. Assoc. 58: 959-960.

Sawtell, N.B., Frenkel, M.Y., Philpot, B.D., Nakazawa, K., Tonegawa, S. and Bear, M.F. 2003. NMDA receptor-dependent ocular dominance plasticity in adult visual cortex. Neuron 38: 977-985.

Scheiman, M.M., Hertle, R.W., Beck, R.W., Edwards, A.R., Birch, E., Cotter, S.A., Crouch Jr., E.R., Cruz, O.A., Davitt, B.V., Donahue, S., et al. 2005. Randomized trial of treatment of amblyopia in children aged 7 to 17 years. Arch. Ophthalmol. 123: 437-447.

Selenow, A. and Ciuffreda, K.J. 1986. Vision function recovery during orthoptic therapy in an adult esotropic amblyope. J. Am. Optom. Assoc. 57: 132-140.

Shatz, C.J. and Stryker, M.P. 1978. Ocular dominance in layer IV of the cat's visual cortex and the effects of monocular deprivation. $J$. Physiol. 281: 267-283.

Skibinska, A., Glazewski, S., Fox, K., and Kossut, M. 2000. Age-dependent response of the mouse barrel cortex to sensory deprivation: A 2-deoxyglucose study. Exp. Brain Res. 132: 134-138.

Smith, D.C. 1981. Functional restoration of vision in the cat after long-term monocular deprivation. Science 213: 1137-1139.

Smith, D.C., Holdefer, R.N., and Reeves, T.M. 1982. The visual field in 
monocularly deprived cats and its permanence. Behav. Brain Res. 5: 245-259.

Tagawa, Y., Kanold, P.O., Majdan, M., and Shatz, C.J. 2005. Multiple periods of functional ocular dominance plasticity in mouse visual cortex. Nat. Neurosci. 8: 380-388.

Taha, S. and Stryker, M.P. 2002. Rapid ocular dominance plasticity requires cortical but not geniculate protein synthesis. Neuron 34: $425-436$

Tyler, C.W., Apkarian, P., Levi, D.M., and Nakayama, K. 1979. Rapid assessment of visual function: an electronic sweep technique for the pattern visual evoked potential. Invest. Ophthalmol. Vis. Sci. 18: $703-713$.
Wick, B., Wingard, M., Cotter, S., and Scheiman, M. 1992. Anisometropic amblyopia: Is the patient ever too old to treat? Optom. Vis. Sci. 69: 866-878.

Wiesel, T.N. and Hubel, D.H. 1963. Single-cell responses in striate cortex of kittens deprived of vision in one eye. J. Neurophysiol. 26: $1003-1017$.

Wiesel, T.N. and Hubel, D.H. 1965. Extent of recovery from the effects of visual deprivation in kittens. J. Neurophysiol. 28: 1060-1072.

Received June 20, 2007; accepted in revised form July 9, 2007. 


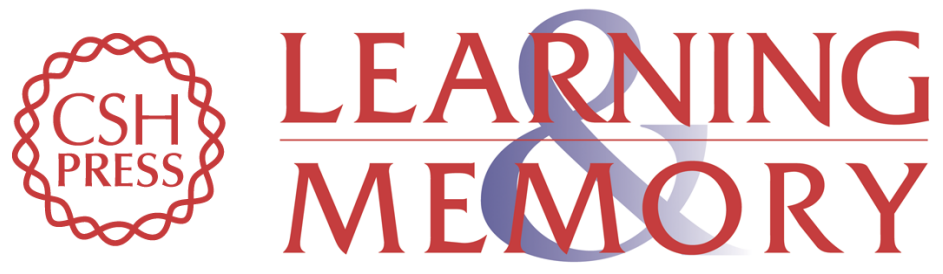

\section{Adult visual experience promotes recovery of primary visual cortex from long-term monocular deprivation}

Quentin S. Fischer, Salman Aleem, Hongyi Zhou, et al.

Learn. Mem. 2007, 14:

Access the most recent version at doi:10.1101/lm.676707

References This article cites 64 articles, 17 of which can be accessed free at:

http://learnmem.cshlp.org/content/14/9/573.full.html\#ref-list-1

License

Email Alerting Receive free email alerts when new articles cite this article - sign up in the box at the Service top right corner of the article or click here. 